Archivo de casos del Instituto Nacional de Toxicología.

Departamento de Sevilla.

\title{
Muerte inesperada súbita en el Servicio de Urgencias, de paciente tratada por neurosis de ansiedad, portadora de un feocromocitoma descubierto durante la autopsia. Sudden unexpected death at the medical Emergency Service of a patient, treated for anxiety neurosis, who had a heochromocytoma, found at forensic autopsy.
}

\section{A. Serratosa1, F. Sánchez² y A. Garfia ${ }^{3}$}

\section{RESUMEN}

Los pacientes que sufren problemas psiquiátricos graves, y aquellos otros cuya sintomatología se encuentra a caballo entre los trastornos emocionales puros y los dependientes de la somatización de los conflictos psíquicos constituyen un colectivo de enfermos que necesitan de especial atención y esfuerzo diagnóstico. Los primeros conforman un grupo de alto riesgo de muerte inesperada por enfermedades, tan comunes, que realmente sorprende que no fuesen diagnosticadas y tratadas a tiempo. Los segundos se enfrentan a la dificultad diagnóstica de diferenciar entre lo verdaderamente emocional y lo somático, con el peligro secundario de obviar la verdadera causa somática de algunos cuadros clínicos con sintomatología fácilmente etiquetada de psíquica. A veces la realidad se impone de manera drástica, y legalmente conflictiva para el médico o el equipo de asistencia hospitalaria. Presentamos un caso de muerte inesperada, que tuvo lugar en el Servicio de Urgencias, de una paciente diagnosticada de neurosis de ansiedad. La Autopsia Médico-Legal descubrió la existencia de un feocromocitoma de la médula suprarrenal izquierda.

Palabras clave: Muerte inesperada súbita. Neurosis de ansiedad. Feocromocitoma. Autopsia Médico-Legal. Malapraxis.

\section{ABSTRACT}

Patients who suffer serious psychiatric problems, and those others whose symptoms are half way between purely emotional disorders and those dependent on somatization of psychological conflicts, make up a group of patients for whom diagnosis requires special attention and effort. The first form a group at high risk of unexpected death from illnesses so common that it is really surprising that they are not diagnosed and treated in time. The second are faced with the difficulty at diagnosis of differentiating between the truly emotional and the somatic, with the secondary danger of obviating the real somatic cause of some clinical state, with symptoms easily labelled as psychological. At times reality asserts itself drastically, and is legally conflictive for the doctor or the hospital team. We present a case of unexpected death, which took place at the Medical Emergency Service, of a patient diagnosed with anxiety neurosis. The forensic autopsy discovered the existence of a pheochromocytoma of the left suprarenal medulla.

Key words: Sudden unexpected death. Anxiety neurosis. Pheochromocytoma. Forensic autopsy. Medical malpractice.

\footnotetext{
Correspondencia: Dr. A.Garfia. e-mail: a.garfiaggg@teleline.es.

1 Médico Forense de Lebrija (Sevilla).

2 Médico Forense de Badajoz.

3 Jefe del Servicio de Anatomía Patológica. INT. Sevilla. Prof. Titular.
} 


\section{INTRODUCCIÓN}

Con extremada frecuencia acuden a un Servicio de Urgencias pacientes que presentan sintomatología orgánica inespecífica y un intenso componente ansioso. Estos cuadros son diagnosticados -la mayor parte de las veces- de trastornos emocionales agudos, y tratados como tales. En ocasiones, sin embargo, se produce un desenlace fatal, sin causa aparentemente relacionada con la gravedad del cuadro clínico, que sorprende al equipo médico asistencial por su rápida e inesperada presentación. La muerte, en estos casos, no solo se presenta como algo inesperado para médicos y familiares del paciente sino que, habitualmente, desencadena un proceso judicial tras la correspondiente denuncia familiar por malapraxis.

Las llamadas "muertes súbitas verdaderas" suelen ocurrir en un espacio de tiempo muy corto desde el comienzo de los síntomas hasta el desenlace fatal. Las denominadas "muertes inesperadas" pueden ser súbitas (generalmente antes de la primera hora del comienzo de los síntomas o del shock, y suelen ser observadas), o prolongadas (la muerte ocurre durante las primeras veinticuatro horas y son, generalmente, hospitalarias). En ambas categorías, los órganos deberían ser examinados en el siguiente orden:

I.- El corazón (arterias coronarias).

2.- Los pulmones y las arterias pulmonares.

3.- El cerebro.

4.- La laringe y la tráquea.

5.- El resto de los órganos (aorta, hígado, riñón, etc.).

En un $95 \%$ de los casos de muerte súbita verdadera y en la mayoría de los casos de muerte inesperada - súbita o prolongada- las lesiones causantes de la muerte se encuentran en uno de los cinco órganos citados, por ese orden. Cuando la causa de la muerte no se encuentra en uno de los tres primeros, nos encontraremos ante un caso difícil [I].

Podemos afirmar, de una manera general, que las principales lesiones patológicas causantes de muerte súbita son:

\section{Cardiacas}

I. I Fibrilación ventricular

Arteriosclerosis coronaria grave de tres troncos.

Trombosis coronaria

Otras (miocarditis, estenosis aórtica, miocardiopatías, etc.).

I.2 Ruptura cardiaca post-infarto de miocardio.

\section{Pulmonares}

2.I Tromboembolismo pulmonar.

2.2 Embolia gaseosa.

\section{Cerebrales}

2.3 Hemorragia pulmonar masiva por diabrosis (TBC, tumores).

3.I Hemorragia subaracnoidea, o cerebral con inundación ventricular.

3.2 Hemorragia cerebelosa

\section{Laríngeas}

4.I Obstrucción por cuerpos extraños.

4.2 Edema laríngeo por anafilaxia.

\section{Aórticas}

5.I Aneurisma disecante con hemopericardias.

5.2 Colapso coronario e I.A.M. por disección retrógrada. 
Muchos de los casos de muerte inesperada [I] ocurren entre personas que presentan una patología similar a la que se observa en la práctica hospitalaria rutinaria. Las lesiones encontradas en la autopsia indican que la mayor parte de estos pacientes son portadores de enfermedades crónicas mal atendidas que debieron presentar empeoramiento progresivo, sin asistencia médica, o un alto umbral para el dolor y el sufrimiento físico, que viven solos o son pacientes psiquiátricos. Por eso, las lesiones más frecuentemente encontradas en los casos de muerte inesperada son, las siguientes: cardiacas (insuficiencia cardiaca congestiva de diferentes etiologías); pulmonares (neumonía, neumopatía obstructiva crónica, tuberculosis); nerviosas (infarto y hemorragia cerebral, meningitis, tumores cerebrales, encefalopatía aguda de Wernicke); gastrointestinales (ulcus sangrante/perforado, varices esofágicas, pancreatitis, obstrucción intestinal); genitourinarias (pielonefritis aguda con septicemia, riñón terminal).

Los casos de muerte inesperada asociados a la patología tumoral son más bien escasos. En general, los tumores pueden causar muerte súbita verdadera cuando provocan trastornos circulatorios graves (tales como hemorragias cerebrales masivas secundarias a trastornos hematológicos -leucemias- o hemoptisis mortales, por invasión vascular tumoral y diabrosis de la pared de las arterias pulmonares); tromboembolismo pulmonar masivo y/o embolización pulmonar tumoral (cánceres hepáticos y del aparato digestivo); o carcinomatosis masiva (cuyos tumores primarios se encuentran en la mama, en el aparato digestivo, páncreas y órganos genitales). Otros tumores, como el que nos ocupa en este trabajo -el feocromocitoma- pueden producir patología cardio-vascular grave, incluyendo muerte súbita, debido a la producción, en exceso, de mensajeros químicos que actúan sobre-estimulando el sistema cardiovascular o provocando una miocarditis aguda norepinefrínica tóxica $[\mathrm{l}$ I], por un efecto tóxico directo de las catecolaminas sobre los miocardiocitos. Entre estos mensajeros se encuentran: la adrenalina, la noradrenalina y el péptido denominado adrenomedulina, el cual posee un potente efecto vasodilatador, natriurético y diurético [2].

Presentamos un caso de muerte inesperada, que tuvo lugar en el Servicio de Urgencias, de una paciente diagnosticada de neurosis de ansiedad que estaba siendo tratada con ansiolíticos. La Autopsia Médico-Legal descubrió la existencia de una tumoración de $7 \times 5 \times 3 \mathrm{~cm}$, localizada sobre el riñón izquierdo. El estudio histopatológico, realizado en el Servicio de Anatomía Patológica del Instituto Nacional de Toxicología de Sevilla, demostró que se trataba de un feocromocitoma.

Pretendemos llamar la atención sobre la frecuencia con que la ansiedad y otros síntomas emocionales entran a formar parte del cortejo sintomático de enfermedades somáticas graves, a veces fatales, e incidir en la gran utilidad de la autopsia como herramienta indispensable para lograr un diagnóstico certero de la causa de la muerte. En pleno siglo XXI, donde las técnicas de diagnóstico por imagen han alcanzado un grado tal de perfección que han conseguido que los cuerpos sean "cuasi-transparentes", una autopsia reglada continúa siendo un eficaz método de estudio de la enfermedad y de la causa de la muerte, en numerosas ocasiones. La Patología Forense y la Autopsia Médico-Legal ofrecen, cada día, una fuente inagotable de conocimiento sobre la enfermedad y sobre la causa y la manera de la muerte, a pesar de los espectaculares avances tecnológicos aplicados en el denominado diagnóstico por la imagen.

\section{DESCRIPCION DEL CASO}

\section{ANTECEDENTES PATOLÓGICOS:}

Mujer de 50 años de edad, hipertensa, y diabética no insulinodependiente de diagnóstico reciente. Había sido asistida varias veces en los Servicios de Urgencias del Hospital por crisis de "ansiedad", acompañadas de dolor precordial, a veces acompañado de episodios de taquicardia. Los 
estudios electrocardiográfico y bioquímico enzimático se habían considerado, siempre, dentro de la normalidad. En la actualidad se encontraba en tratamiento con medicación ansiolítica por su "neurosis de ansiedad".

\section{CirCunstancias de LA MUERTE:}

El día del óbito acudió a Urgencias, sobre las 3 horas de la madrugada, aquejada de dolor epigástrico, disnea y vómitos teñidos de color rojizo. La TA era de I20/60 y el ECG presentaba ritmo sinusal a 120 x '. Después de la colocación de una sonda nasogástrica se objetivó un líquido claro con algún resto hemático. Pasó a observación presentando un aumento de su disnea con gasometría PH 7.21, $\mathrm{PCO}_{2} 33, \mathrm{PO}_{2}$ 50, $\mathrm{HCO}_{3}-13, \mathrm{O}_{2}$ SAT 77\%, y dos episodios de bradicardia -seguidos de parada cardiorrespiratoria - de la que salió con maniobras de RCP. Ingresó en UCl a las 4.30 horas, siendo conectada a ventilación mecánica, y presentando una TA 60/0; ECG en ritmo sinusal a 130 x '; Glasgow de 3; y, midriasis arreactiva. Permaneció en anuria, acentuándose la inestabilidad hemodinámica y entrando, finalmente, en PCR. Fallece a las 7 horas, de ese mismo día, con el diagnóstico de shock cardiogénico y edema agudo de pulmón. Ante la negativa familiar a la necropsia clínica, se llevó a cabo una autopsia judicial y estudios anatomopatológicos por el Instituto Nacional de Toxicología.

\section{ESTUDIO NECRÓPSICO}

\section{EXAMEN EXTERNO:}

Cadáver de una mujer de biotipo leptosomático, de 50 años de edad y $158 \mathrm{~cm}$ de talla. Presenta un abdomen globuloso y se observa la presencia de hongo espumoso por la boca y los orificios nasales. Se objetivan signos de actuación médica reciente, consistentes en punturas en la región clavicular derecha, ambos antebrazos y regiones inguinales; todas ellas se encuentran cubiertas con apósitos.

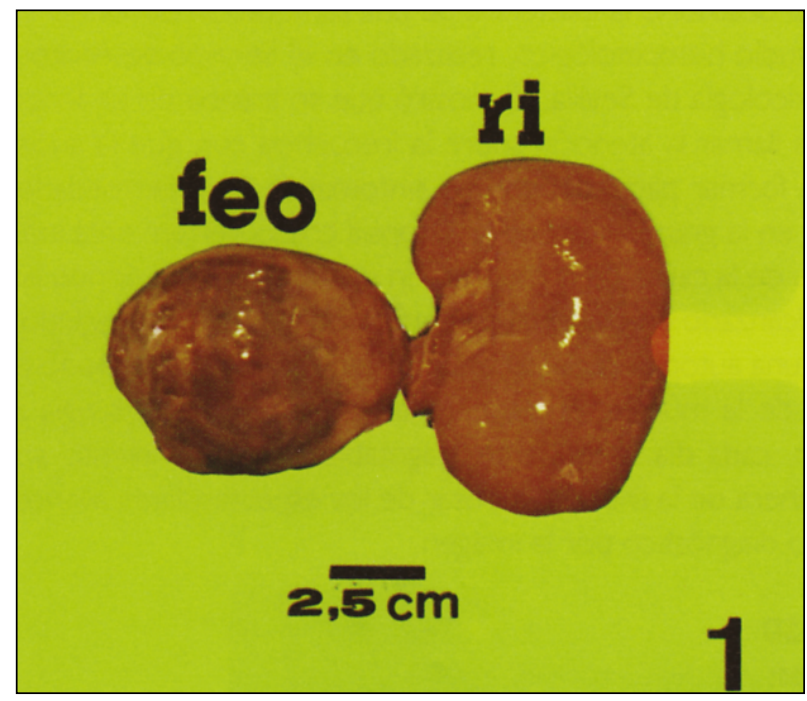

A Foto $\mathrm{n}^{\circ}$ 1.- Aspecto macroscópico del tumor (feo= feocromocitoma), ocupando la fosa renal izquierda (ri= riñón izquierdo). 


\section{EXAMEN INTERNO:}

- Cavidad craneana: áreas de extravasación hemática en el espacio subaracnoideo, en el lóbulo parietal izquierdo y en el hemisferio cerebeloso del mismo lado.

- Cuello: escoliosis de columna cervical.

- Tórax: pulmones pesados, húmedos y congestivos que rezuman abundante líquido sero-sanguinolento a la expresión ligera. El corazón, de 300 g, post-fijación, presentaba los siguientes parámetros macroscópicos: VT $9.0 \mathrm{~cm}$. VP $6.0 \mathrm{~cm}$. VM $7.0 \mathrm{~cm}$. VA $6.0 \mathrm{~cm}$. Pared ventricular derecha $0.4 \mathrm{~cm}$. Pared ventricular izquierda $1.6 \mathrm{~cm}$, con discreta dilatación ventricular.

- Abdomen: hígado congestivo. Erosiones leves en la mucosa gástrica, en la región fúndica. En la celda renal izquierda se detectó una masa pararrenal, redondeada, de 7×5×3 cm de diámetros máximos (Fig. I); presentaba, al corte, coloración marrón oscura alternante con áreas hemorrágicas y otras de coloración blanquecina. Ambos riñones eran de aspecto, tamaño y peso normal. Los ovarios eran poliquísticos y presentaban, al corte, algunas calcificaciones.

Se enviaron, al Servicio de Patología del Instituto de Toxicología, muestras de cerebro, cerebelo, pulmón, riñón, el corazón y la tumoración descrita arriba.

\section{ESTUdIO HISTOPATOLÓGICO:}

- Encéfalo: congestión meníngea. Hemorragia subaracnoidea cerebelosa, focal.

- Corazón: hiperplasia fibromuscular concéntrica, de la íntima arterial de las arterias coronarias principales -de tipo hipertensivo- de grado moderado (50-75 \% de estenosis luminal). Marcada a severa arteriolosclerosis hipertensiva de las arteriolas epicárdicas e intramurales (Fig. 2) . Miocardiocitos que presentan necrosis en bandas de contracción en los músculos papilares ventriculares izquierdos y en la pared libre del ventrículo derecho (Fig. 3). Placas de fibrosis endocárdica en el ventrículo izquierdo que se extienden por el seno de los músculos papilares anterolateral y posterior.

- Pulmón: congestión y edema alveolar submasivo.

- Riñón: congestión córtico-medular. Arteriolosclerosis hipertensiva, de grado moderado. Fibroma medular de I mm de diámetro que no presenta signos de malignidad.

- Masa tumoral de fosa renal: tumoración constituida por nidos de células poligonales, de tamaño variable, las mayores de las cuales presentan un amplio citoplasma basófilo, ocupado por granulaciones groseras acidófilas. Los núcleos son redondeados y, a veces, se encuentran nidos de células pleomórficas, con núcleos gigantes y abigarrados. En el seno del tumor se encuentran algunas células ganglionares, así como restos histológicos de la corteza suprarrenal. La tumoración se encuentra rodeada por una cápsula de tejido conjuntivo fibroso denso aunque, en el seno de ella, se encuentran algunas células tumorales. (Fig. 4 y 5 ).

Se emitieron los siguientes diagnósticos patológicos:

- Encéfalo.- Hemorragia subaracnoidea focal.

- Corazón.- Cardiomegalia discreta con dilatación ventricular izquierda. Arterio y arteriolosclerosis hipertensiva. Miocardiopatía isquémica crónica. Miocarditis aguda tóxica norepinefrínica ( Miocardiopatía catecolamínica).

- Pulmón.- Edema pulmonar submasivo.

- Riñones.- Nefropatía hipertensiva. Fibroma medular de $1 \mathrm{~mm}$.

- Tumor de fosa renal.- Feocromocitoma suprarrenal (Paraganglioma suprarrenal). 
Muerte inesperada súbita en el servicio de urgencias, de paciente tratada por neurosis de ansiedad, portadora de un feocromocitoma descubierto durante la autopsia.
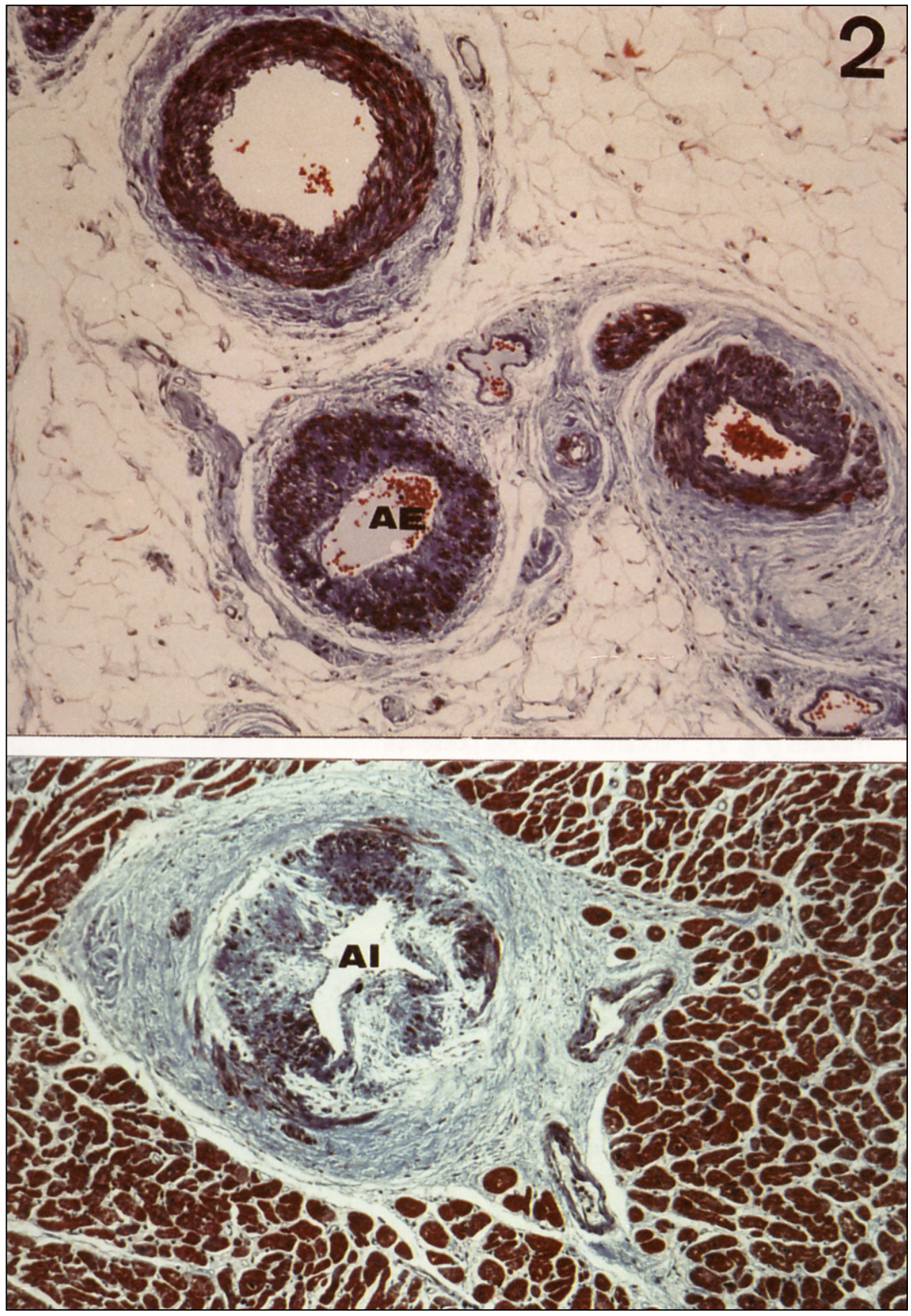

$\Delta$ Foto $\mathbf{n}^{\circ}$ 2.- Muestra cambios hipertensivos de la capa media de las arteriolas epicárdicas (AE) y de las arteriolas intramurales de la pared libre ventricular izquierda y del tabique interventricular (Al). Tricrómico de Masson. $120 X$. 


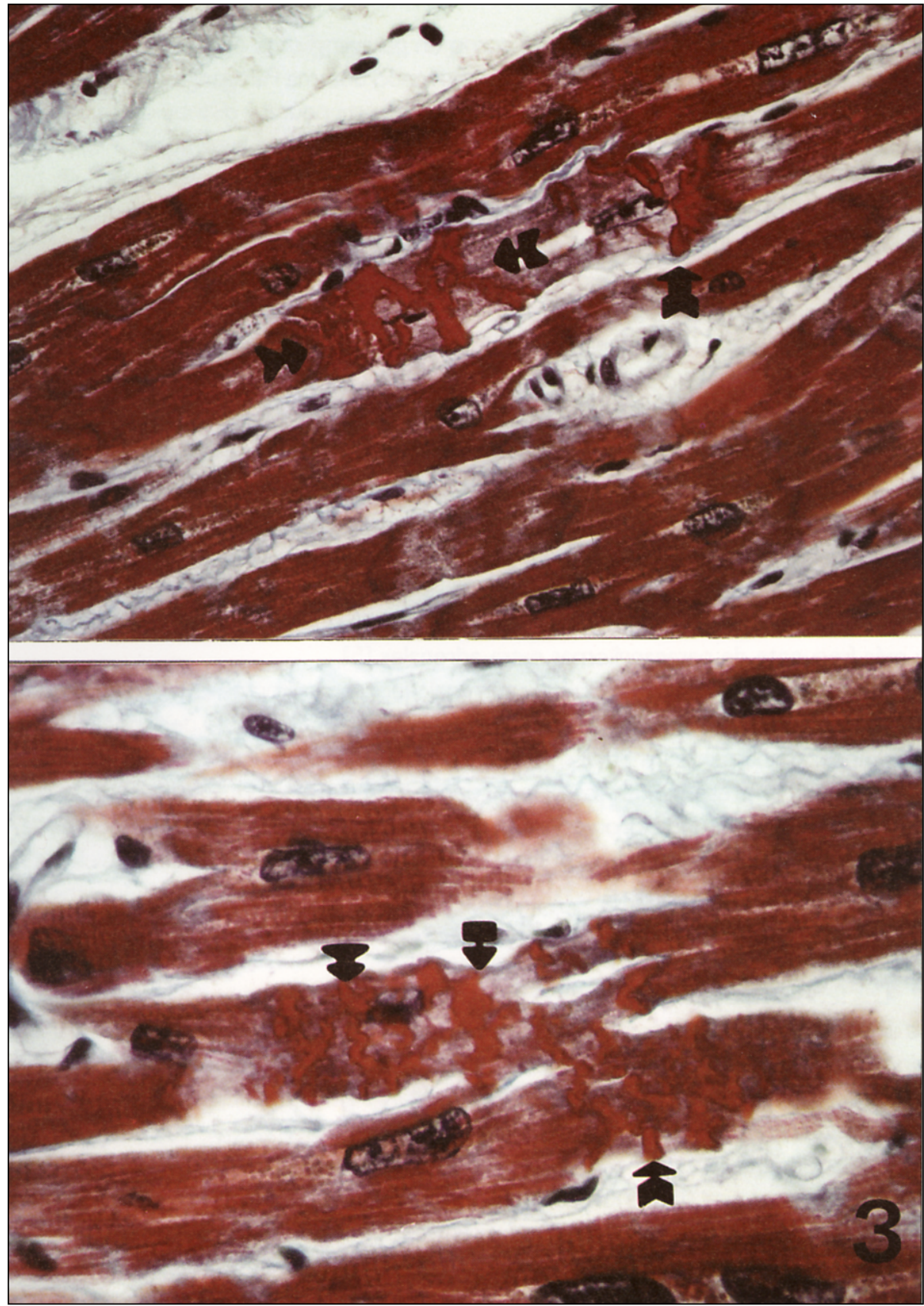

$\Delta$ Foto $n^{\circ}$ 3.- Miocarditis epinefrínica (catecolamínica). Miocardiocitos aislados presentan necrosis en bandas de contracción (flechas), el daño miocárdico agudo más precoz distinguible microscópicamente, de distribución multifocal, no vásculo-dependiente. 480 y 756 X. Tricrómico e Masson. 


\section{DISCUSIÓN}

El feocromocitoma puede definirse como un paraganglioma de la médula suprarrenal. Los paragangliomas son tumores originados en el denominado Sistema Paraganglionar el cual se encuentra formado por colecciones de células, originadas en la cresta neural y distribuidas por todo el organismo, que incluye, entre otros, a los cuerpos o glomus carotídeos y aórticos [3, 4, 5]. La característica morfológica común de todas estas colecciones celulares es la presencia, en su citoplasma, de gránulos neurosecretores que contienen catecolaminas. El miembro más significativo del Sistema Paraganglionar es la médula suprarrenal (un sistema neuroefector conectado con el sistema nervioso ortosimpático). Los paraganglios extra-adrenales se pueden dividir en dos grandes categorías: los relacionados con el Sistema Parasimpático (noveno y décimo pares craneales, y posiblemente, el tercero y el quinto par), y los conectados con el Sistema Ortosimpático. Los primeros son, generalmente, no cromafines (no dan positiva la reacción cromafin, descrita por Henle en I84I y I865, consistente en la aparición de color pardo cuando la fijación de la médula suprarrenal se realizaba con soluciones de ácido crómico - $\mathrm{CrO}_{4} \mathrm{H}_{2}-$ ), se concentran en la cabeza, el cuello y el mediastino, y poseen propiedades quimiorreceptoras. Los conectados con el Sistema Ortosimpático son cromafines, predominan en el retroperitoneo, a lo largo de las regiones para-aórticas y tóracolumbar y representan, probablemente, los hermanos pequeños de la familia de la médula suprarrenal [5].

Los paragangliomas han sido descritos en, prácticamente, todos los lugares donde existen paraganglios normales. Los paragangliomas del cuerpo carotídeo constituyen el grupo más importante y más frecuente de paragangliomas extra-adrenales [5].

El paraganglioma de la médula suprarrenal -feocromocitoma- es el mejor conocido y el tumor más frecuente de la medula suprarrenal. El feocromocitoma ha sido denominado [8] el tumor del 10\% - aproximadamente un 10\% son malignos; un 10\% son bilaterales; el 10\% son extramedulares; y el 10\% se presentan en los niños y pueden asociarse a un síndrome MEN (Neoplasias Endocrinas Múltiples) o a un síndrome familiar genético, autosómico dominante, como la neurofibromatosis tipo I, la enfermedad de Von Hippel-Lindau y el síndrome de Sturge-Weber [9].

Los feocromocitomas no familiares se desarrollan en adultos, entre los 40 y los 60 años de edad, con discreto predominio femenino. En cambio, los síndromes familiares que se presentan en la infancia, muestran predisposición por los varones.

Los feocromocitomas son tumores poco frecuentes, aunque importantes, porque -al igual que los adenomas corticales secretores de aldosterona- provocan hipertensión arterial que puede corregirse quirúrgicamente. La hipertensión debida a un tumor no diagnosticado puede ser mortal. En un 50\% de los casos, la hipertensión suele presentarse en forma de crisis paroxística que puede desencadenar una insuficiencia cardiaca aguda mortal [ 10$]$, aunque estos pacientes también presentan cambios cardiovasculares dependientes de una hipertensión crónica (véase la Fig. 2), o riesgos de hipotensión ortostática (iadrenomedulina?). Los ataques hipertensivos paroxísticos pueden ser desencadenados por numerosas circunstancias, entre las que se han descrito las siguientes: consumo de determinados fármacos, anestésicos locales, parto, cirugía menor, etc. La triada sintomática de ataques de sudoración, taquicardia y dolor de cabeza es sospechosa de feocromocitoma. La confirmación diagnóstica se realiza mediante el estudio de las catecolaminas urinarias o de sus metabolitos (el ácido vanil mandélico y las metanefrinas) y de la TAC. En estos tumores no existen marcadores morfológicos de malignidad, a excepción de la existencia de metástasis. Los tumores malignos, cuando se dan, tienen una especial predilección a metastatizar en los huesos, especialmente en las costillas y en la columna vertebral; por eso se recomienda realizar un escáner del esqueleto en pacientes sospechosos de padecer un feocromocitoma. 
La muerte inesperada, en estos pacientes, se puede producir tras la aparición de una hipertensión paroxística -debida a la liberación brusca de catecolaminas- que puede desencadenar una insuficiencia cardiaca congestiva aguda.

Las complicaciones asociadas a los feocromocitomas son, fundamentalmente, cardiovasculares. La conocida como miocardiopatía catecolamínica provoca una inestabilidad del miocardio que predispone a la presentación de arritmias ventriculares. Esta vulnerabilidad parece secundaria a alteraciones isquémicas- por vasoconstricción de las arterias coronarias y de las arteriolas intramiocárdicas- que son catecolaminas dependientes; aunque también está demostrado un efecto tóxico directo de las catecolaminas sobre los miocardiocitos de trabajo. La respuesta miocárdica a la toxicidad de las catecolaminas se conoce desde hace bastantes años. La denominada por Karch [ I I] "miocarditis norepinefrínica", se observó casi desde el comienzo de la utilización de las drogas hipertensoras, por vía intravenosa [12]. La lesión miocárdica característica es indistinguible de la que se detecta en el feocromocitoma. Histológicamente, el daño miocárdico lesional agudo más precoz, que se puede distinguir microscópicamente, es la llamada "necrosis en bandas de contracción" de miocardiocitos aislados que se encuentran rodeados de células normales (aunque mejor expresado son bandas de hipercontracción de los miofilamentos de actina y de miosina, con una intensa contracción, acumulación y acercamiento del material contráctil hacia las líneas o bandas Z), de distribución multifocal y no vásculo-dependientes. Este tipo de lesión es similar a la producida en los adictos a la cocaína y a las anfetaminas [I I]. Las lesiones pueden presentarse en cualquier parte del corazón, pero son más frecuentemente localizadas en el subendocardio de la pared libre ventricular izquierda. Macroscópicamente, el peso cardiaco suele estar aumentado [13] y se cree que la hipertrofia miocárdica puede ser secundaria a la hipertensión arterial (lo más probable) o a un efecto tóxico directo catecolamínico sobre el miocardio ( sería un efecto más a largo plazo, derivado de la pérdida progresiva de miocardiocitos con la posterior reparación fibrótica de las "tumbas miocardiocíticas"). Los pacientes con arterias coronarias normales, portadores de un feocromocitoma, pueden sufrir un infarto agudo de miocardio, probablemente secundario a un espasmo arterial [14].

Entre otras complicaciones vasculares importantes del feocromocitoma, se han descrito las siguientes: la estenosis reversible de la arteria renal [15]; la vasoconstricción de las arterias mesentéricas con isquemia intestinal subsidiaria [16]; la disección de las arterias carótidas, secundaria a una crisis hipertensiva [17]; la ruptura de aneurismas cerebrales [18] y la vasculitis leucocitoclástica [19].

Una asociación curiosa ha sido descrita entre el feocromocitoma, el neuroblastoma y el ganglioneuroma, en niños portadores de cardiopatías congénitas cianóticas, sugiriendose por sus autores [20] la existencia de un posible papel hipóxico crónico en el desarrollo de estos tumores.

Decíamos antes que, además de catecolaminas, estos tumores pueden producir otros esteroides -por lo que pueden asociarse a un síndrome de Cushing o a otras endocrinopatías- o un péptido denominado adrenomedulina, el cual posee un potente efecto vasodilatador, diurético y natriurético [2].

\section{AGRADECIMIENTOS}

A la Srta. Teresa Sánchez Botet por su colaboración en el rastreo bibliográfico en Internet y a la Técnica Especalista en Anatomía Patológica, María Luisa Vázquez, por la asistencia técnica. 
Muerte inesperada súbita en el servicio de urgencias, de paciente tratada por neurosis de ansiedad, portadora de un feocromocitoma descubierto durante la autopsia.

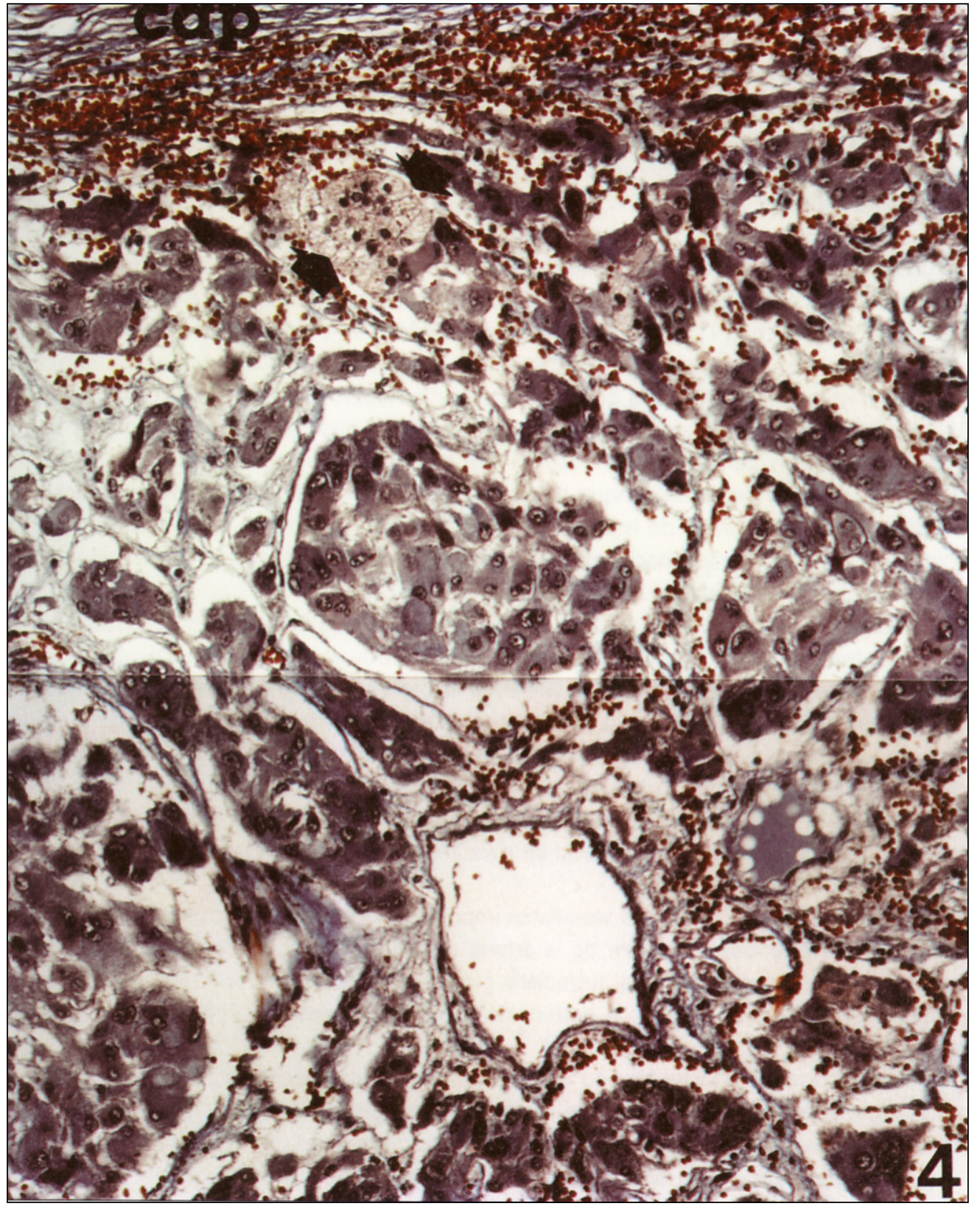

$\Delta$ Foto $n^{\circ}$ 4.- Feocromocitoma. A pequeño aumento las células tumorales se disponen constituyendo nidos y presentando morfología poligonal. Inmediatamente por debajo de la cápsula tumoral (cap), se encontraban restos celulares de la capa glomerulosa de la cápsula suprarrenal (flechas). Tricrómico de Masson. 240 X. 

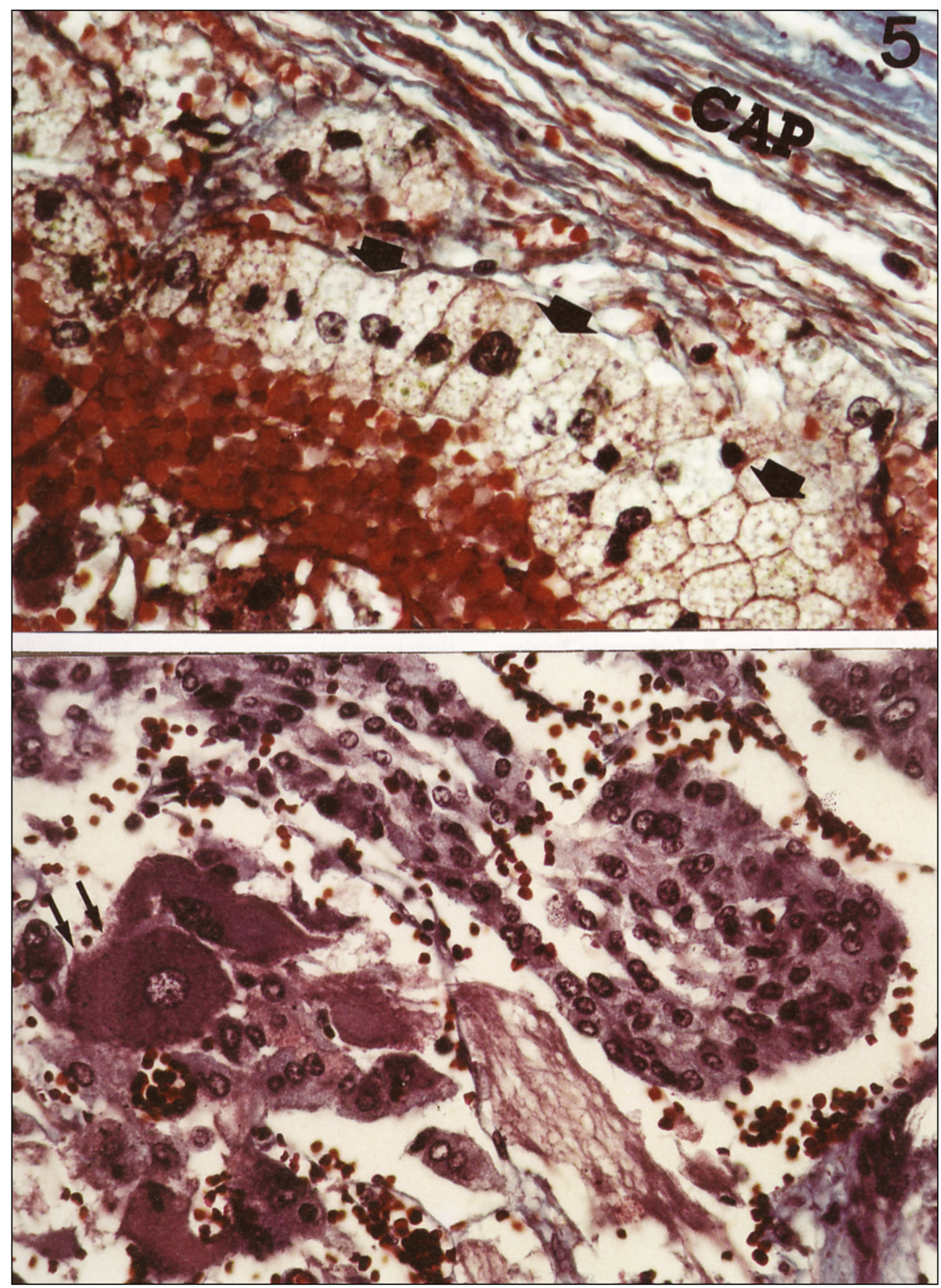

Foto $n^{\circ}$ 5.- Superior. Muestra un detalle de los restos celulares de la corteza suprerrenal (flechas), inmediatamente por debajo de la cápsula tumoral (CAP). $750 \mathrm{X}$.

Inferior.- Nidos de células ganglionares, señaladas por flechas. Tricrómico de Masson. $480 \mathrm{X}$ 


\section{BIBLIOGRAFÍA:}

I. Garfia A, Borondo J.- Muerte súbita en Patología Forense. Rev Esp Med Legal.Enero-Junio 1989; pp. 3I-43.

2. Jougasaki M, Wei C-M, Mckinley LJ.- Elevation of circulatory and ventricular adrenomedullin in human congestive heart failure. Circulation 92: 286-290, 1995.

3. Garfia A.- Glomus tissue in the vicinity of the human carotid sinus. Journal of Anatomy 130: I-I2 (1980).

4. Garfia A.- En Böck, P.- The Paraganglia. Handbuch der mikroskopischen Anatomie. Band 6. Blutgefäß- und Lymphgefäßapparat. 8 Teil.Springer-Verlag. Berlin1982. pp.89.

5. Garfia A.- Muerte Súbita o Retardada asociada a la Patología del Compartimento Lateral del cuello: el papel fisiopatológico de los Barorreceptores del Seno Carotídeo y de los Quimiorreceprores del Glomus Caroticum. Cuad Med For 16-17. 1999. $85-153$

6. Henle J.- Allgemeine Anatomie. Leipzig 1841.

7. Henle J.- Über die Gewebe der Nebenniere und Hypophyse. $Z$ rat Med 24, $143-152$ (1865).

8. Rosai J.- Ackerman's Surgical Pathology. $7^{\text {a }}$ Ed. Ed Mosby C. Washington D.C. 1989.

9. Cotran R, Kumar V, Robbins S.- Pathological Basis of Disease. Interamericana. 1995.

10. Silver MD, Gotlieb Al, Schoen FJ.- Cardiovascular Pathology. Churchill Livingstone.N.Y. 200I.
II. Karch SB .- The Pathology of Drug Abuse. $3^{\mathrm{a}}$ Ed. CRC Press. 2002. pp. 104.

12. Szakacs, J and Cannon, A.- L-Norepinephrine myocarditis. Am J Clin Pathol. 30, pp. 425-434.

13. Van Vliet, Burchell HB, Titus JL.- Focal myocarditis associated with pheochromocytoma. N Engl J Med 274:II02-II05. 1966. 14. Jessurun CR, Adam K, Moisek J Jr, Wilansky S.- Pheochromocytoma-induced myocardial infarction in pregnancy. Tex Heart Inst ] 20:120-124;1993.

15. Pickard JL, Ross G Jr, Silver D.- Coexisting extraadrenal pheochromocytoma and renal artery stenosis: A case report and review of the pathophysiology. J Pediatr Surg 30: 1613-1616. 1995.

16. Morris K, McDevitt B.- Pheochromocytoma presenting as a case of mesenteric vascular occlusion. Ir Med J 78:356-358.1985.

17. Gulliford MC, Hawkins CP, Murphy RP.- Spontaneous dissection of the carotid artery and pheochromocytoma. Br J HospMed 35: $416-420,1986$.

18. DeSouza TG, Berlad L, Shaphiro K et al.,- Pheochromocytoma and multiple intracerebral aneurysms. J Pediatr 108: 947-950. 1986.

19. Kulp-Shorten $\mathrm{CL}$ et al., - Cutaneous vasculitis associated with pheochromocytoma. Arthritis Rheum 33: 1852-1856.1990.

20. De la Monte S, Hutchings GM, Moore GW.- Peripheral neuroblastic tumors and congenital heart disease. Am J Pediatr Hematol Oncol 7: 109-II2. 1985. 